\title{
Type 2 Diabetes, the Metabolic Syndrome and Dyslipidemia in Children and Adolescents
}

\author{
Orit Pinhas-Hamiel \\ Paediatric Endocrinology and Diabetes Unit, Edmond and Lily Safra Children's Hospital, Sheba Medical Center, \\ Tel-Hashomer, Ramat-Gan and Maccabi Juvenile Diabetes Center, Raanana, Sackler School of Medicine, Tel- \\ Aviv University, Israel
}

\begin{abstract}
Understanding risk factors and complications associated with early-onset type 2 diabetes (T2DM) continues to be a major research issue. This year we learn that women with T2DM likely suffered from sexual abuse in their childhood. This is extremely important to remember each time an obese child is evaluated in a clinic. Beyond stopping the current insult, awareness and early intervention may prevent increased weight gain and future morbidity. Regarding the early development of diabetes complications, we learn for the first time about cognitive abnormalities presenting among adolescents with T2DM, even after short disease duration and moderate metabolic control. Prematurity has been added to the long list of risk factors of early-onset T2DM. Contrasting with this long-term effect, a longitudinal study of adolescent males found the risk of diabetes to be mainly associated with increased body mass index (BMI) close to the time of diagnosis, and not to their degree of obesity during adolescence. Finally, two articles suggesting a model for the link between obesity, insulin resistance and chronic inflammation, as well as a possible intervention in the signaling pathway, were published this year.

A newly discovered mechanism elucidates the regulation of the cascade of genes leading to the development of the metabolic syndrome (MetS). The significance of fasting insulin levels as well as the reproducibility of the oral glucose tolerance test in obese children have been challenged. The surprising results of a school intervention program for diabetes risk reduction are presented. A strong association was demonstrated between elevated non-HDL levels and the metabolic syndrome.

The current guidelines for the screening of dyslipidemia are being questioned; in a large cohort study it was found that current practice misses a considerable number of individuals with abnormal LDL levels. This year the Cochrane Library published a review on the safety and benefits of statin treatment in children. In addition, we learn about the effect of maternal hyperlipidemia during pregnancy on fetal programming of hyperlipidemia later in life.
\end{abstract}

\section{Type 2 diabetes in children and adolescents}

Food for thought

\section{Abuse in childhood and adolescence as a predictor of type 2 diabetes in adult women}

Rich-Edwards JW, Spiegelman D, Lividoti Hibert EN, Jun HJ, Todd TJ, Kawachi I, Wright RJ

Connors Center for Women's Health and Gender Biology, Brigham and Women's Hospital/Harvard Medical School, Boston, Mass., USA.

jr33@partners.org

Am J Prev Med 2010;39:529-36.

Background: Although child abuse is associated with obesity, it is not known whether early abuse increases risk of type 2 diabetes (T2DM). The authors aimed to investigate associations of child and adolescent abuse with T2DM.

Methods: Proportional hazard models were used to examine associations of lifetime abuse reported in 2001 with risk of diabetes from 1989 to 2005 among 67,853 women in the Nurses' Health Study II. Data were analyzed in 2009. 
Results: Child or teen physical abuse was reported by $54 \%$ and sexual abuse by $34 \%$ of participants. Models were adjusted for age, race, body type at age 5 years, and parental education and history of diabetes. Compared to women who reported no physical abuse, the hazards ratio (HR) was $1.03(95 \%$ $\mathrm{CI}=0.91,1.17)$ for mild physical abuse, $1.26(1.14,1.40)$ for moderate physical abuse, and $1.54(1.34$, 1.77) for severe physical abuse. Compared with women reporting no sexual abuse in childhood or adolescence, the HR was $1.16(95 \% \mathrm{CI}=1.05,1.29)$ for unwanted sexual touching, $1.34(1.13,1.59)$ for one episode of forced sexual activity, and $1.69(1.45,1.97)$ for repeated forced sex. Adult BMI accounted for $60 \%(95 \% \mathrm{CI}=32 \%, 87 \%)$ of the association of child and adolescent physical abuse and $64 \%$ $(95 \% \mathrm{CI}=38 \%, 91 \%)$ of the association of sexual abuse with diabetes.

Conclusions: Moderate to severe physical and sexual abuse in childhood and adolescence have doseresponse associations with risk of T2DM among adult women. This excess risk is partially explained by the higher BMI of women with a history of early abuse.

Data from a national survey conducted in the United States revealed that $17.6 \%$ of women and $3 \%$ of men had been victims of a completed or attempted rape at some time in their lives. Among female victims, $21.6 \%$ were younger than age 12 when they were first raped, and $32.4 \%$ were aged $12-17$ [1].

The association between childhood sexual abuse and obesity has been documented. Of a sample of morbidly obese adults, candidates for bariatric surgery, about one third reported sexual abuse, a rate 2-3 times higher than reported in normative community samples. The relationship between childhood sexual abuse and adult obesity appears to increase with the severity of the abuse. In a longitudinal prospective study, the BMI of girls who were sexually abused between ages 6 and 16 years increased more steeply until age 21 than did the BMI of those who were not abused. Those abused were more likely to be obese at this age [2].

There are several possible explanations for the observed associations between obesity and childhood sexual abuse. Firstly, stressful conditions have been associated with increased risk of obesity. Secondly, it has been suggested that obesity may serve as an 'adaptive function' in which individuals who have been abused try to 'de-sexualize' themselves as a means of protection. Finally, binge eating disorders are associated with both sexual abuse and obesity. Women who reported childhood physical abuse had twice the odds of suffering from an eating disorder and women who reported both physical and sexual abuse during childhood had nearly a fourfold increase. Binge eating disorders are reported to be at least 6 times more common in obese individuals as in normal weight ones. Possible mediators between abuse and disordered eating include poor self-esteem, body image disturbance, behavioral impulsivity, and drug use.

In the current study the authors hypothesized a direct association between physical and sexual abuse and the risk of T2DM. One third of the participants reported sexual abuse in childhood. While mild physical abuse was not associated with an increased risk for T2DM, moderate and severe physical abuse were associated with 26 and $54 \%$ increased risks of diabetes respectively. Women who experienced forced sex once or repeatedly had $34-69 \%$ increased risks of diabetes. Adult BMI was an important mediator of the association between early abuse and diabetes. The upshot of these findings is that the occurrence of sexual abuse should be considered in the clinical evaluation of obese children.

\section{New concerns}

\section{Preliminary evidence for brain complications in obese adolescents with type 2 diabetes mellitus}

Yau PL, Javier DC, Ryan CM, Tsui WH, Ardekani BA, Ten S, Convit A

Department of Psychiatry, New York University School of Medicine, New York, N.Y., USA.

Diabetologia 2010;53:2298-306.

Background: Central nervous system abnormalities, including cognitive and brain impairments, have been documented in adults with T2DM who also have multiple comorbid disorders that could contribute to 
these observations. Assessing adolescents with T2DM will allow the evaluation of whether diabetes per se may adversely affect brain function and structure years before clinically significant vascular disease develops.

Methods: 18 obese adolescents with T2DM and 18 obese controls without evidence of marked insulin resistance, matched on age, sex, school grade, ethnicity, socioeconomic status, BMI and waist circumference, completed MRI and neuropsychological evaluations.

Results: Adolescents with T2DM performed consistently worse in all cognitive domains assessed, with the difference reaching statistical significance for estimated intellectual functioning, verbal memory and psychomotor efficiency. There were statistical trends for executive function, reading and spelling. MRIbased automated brain structural analyses revealed both reduced white matter volume and enlarged cerebrospinal fluid space in the whole brain and the frontal lobe in particular, but there was no obvious grey matter volume reduction. In addition, assessments using diffusion tensor imaging revealed reduced white and grey matter microstructural integrity.

Conclusions: Obese adolescents with T2DM had brain abnormalities relative to obese adolescent controls. These abnormalities are not likely to result from education or socioeconomic bias and may result from a combination of subtle vascular changes, glucose and lipid metabolism abnormalities and subtle differences in adiposity in the absence of clinically significant vascular disease.

This is the first study of brain abnormalities in obese adolescents with T2DM. Among adults with T2DM, the increased risk of cognitive impairments and structural brain abnormalities are well established [2]. Interestingly, it has been suggested that Alzheimer's disease may be caused by a deficiency in brain insulin. Moreover, in light of the apparent overlap between T2DM and dementia, Alzheimer's disease has been classified as a neuroendocrine disorder, coined type 3 diabetes, and not only as a neurologic disorder [3]. The importance of the current study is its exploration of young patients who do not have longstanding multiple comorbidities, thus elucidating the effect of diabetes per se on brain function. Using sensitive MRI techniques and neuropsychological testing, 18 adolescents with obesity and T2DM were compared to 18 obese adolescents without signs of insulin resistance. The duration of T2DM was between 5 months and 7.5 years (mean $2.6 \pm 1.9$ ) years, and the mean HbA1C level $8.3 \%$. Despite the relatively short duration of T2DM, their young age (mean $16.5 \pm 1.9$ ), their intermediate metabolic control, and the absence of clinically significant vascular disease, obese adolescents with T2DM had whole brain volume reduction and significantly poorer performance in three domains: estimated intellectual functioning, verbal memory, and psychomotor efficiency. There was no grey matter volume reduction. The authors suggest that these changes may result from a combination of functional vascular changes, hyperglycemia, and lipid metabolism abnormalities. It is also possible that subtle changes already began during the preclinical stage. As suggested by the authors, further investigations are needed, with larger samples, including comparisons between normal weight adolescents and those with T2DM, to elucidate underlying mechanisms. As Hercule Poirot the detective created by Agatha Christie used to say 'It is the little grey cells, mon ami, on which one must rely.'

\section{Preterm birth - a risk factor for type 2 diabetes? The Helsinki Birth Cohort Study}

Kajantie E, Osmond C, Barker DJ, Eriksson JG

Department of Chronic Disease Prevention, National Institute for Health and Welfare, Helsinki, Finland. eero.kajantie@helsinki.fi

Diabetes Care 2010;33:2623-5.

Background: The association between low birth weight and T2DM is well established. The authors studied whether preterm birth carries a similar risk.

Methods: The Helsinki Birth Cohort includes 13,345 men and women born between 1934 and 1944. Of these, 12,813 had adequate data on length of gestation, which we linked with data on special reimbursement for diabetes medication.

Results: Of the subjects, $5.1 \%$ had received special reimbursement after age 40 . In subjects born before 35 weeks of gestation, the odds ratio for diabetes was 1.68 (95\% CI 1.06-2.65) compared with that in those born at term. After adjustment for birth weight relative to length of gestation, the odds ratio was $1.59(1.00-2.52)$. 
Conclusions: Preterm birth before 35 weeks of gestation is associated with an increased risk of T2DM in adult life. The risk is independent of that associated with slow fetal growth.

The list of risk factors for the early development of T2DM includes obesity, family history of T2DM, intrauterine growth retardation, increased maternal weight during pregnancy, maternal T2DM during pregnancy, and treatment with atypical antipsychotic drugs. While the association between low birth weight and the risk of T2DM is well known, less is known about a possible association between prematurity and T2DM. The current study shows birth before 35 weeks of gestation to increase the risk for developing T2DM in later life. Of 12,813 individuals born in the 1930s, 652 (5\%) developed T2DM after the age of 40 years. After adjusting for birth weight, the risk for premature birth was 1.6 times that of birth at term. After adjustment for maternal BMI in late pregnancy, the risk was even higher, 1.72 (95\% Cl 1.03-1.69). Data regarding the week of birth were not available, and thus the risk of extreme prematurity could not be determined.

Reduction in insulin sensitivity has been documented in children born prematurely [4]. To understand the etiology of this insulin resistance, infant nutrition and its influence on insulin sensitivity and postnatal growth were studied in children born at $\leq 32$ weeks' gestation [5]. Greater childhood weight gain was shown to be associated with increased insulin resistance. Further, it was speculated that a high carbohydrate neonatal diet may lead to greater weight gain and a greater reduction in insulin sensitivity, the latter increasing the risk for T2DM.

Incidence rates of premature birth range from 5.5 to $11.4 \%$ in Europe, and $12 \%$ in the United States. A high incidence of premature birth, together with high survival rates, may contribute to the epidemic of T2DM.

\section{Important for clinical practice}

\section{Adolescent BMI trajectory and risk of diabetes versus coronary disease}

Tirosh A, Shai I, Afek A, Dubnov-Raz G, Ayalon N, Gordon B, Derazne E, Tzur D, Shamis A, Vinker S, Rudich A Division of Endocrinology, Diabetes, and Hypertension, Brigham and Women's Hospital, Harvard Medical School, Boston, Mass., USA. atirosh@partners.org

N Engl J Med 2011;364:1315-25.

Background: The association of body mass index (BMI) from adolescence to adulthood with obesityrelated diseases in young adults has not been completely delineated.

Methods: A prospective study in which 37,674 apparently healthy young men were followed for incident angiography-proven coronary heart disease and diabetes through the Staff Periodic Examination Center of the Israeli Army Medical Corps. The height and weight of participants were measured at regular intervals, with the first measurements taken when they were 17 years of age.

Results: During approximately 650,000 person-years of follow-up (mean follow-up 17.4 years), we documented 1,173 incident cases of type 2 diabetes and 327 of coronary heart disease. In multivariate models adjusted for age, family history, blood pressure, lifestyle factors, and biomarkers in blood, elevated adolescent BMI (the weight in kilograms divided by the square of the height in meters; mean range for the first through last deciles 17.3-27.6) was a significant predictor of both diabetes (hazard ratio for the highest vs. the lowest decile, 2.76; 95\% confidence interval (CI), 2.11-3.58) and angiographyproven coronary heart disease (hazard ratio 5.43; 95\% CI 2.77-10.62). Further adjustment for BMI at adulthood completely ablated the association of adolescent BMI with diabetes (hazard ratio 1.01; 95\% CI $0.75-1.37$ ) but not the association with coronary heart disease (hazard ratio $6.85 ; 95 \%$ CI 3.30 14.21). After adjustment of the BMI values as continuous variables in multivariate models, only elevated $\mathrm{BMI}$ in adulthood was significantly associated with diabetes $(\beta=1.115, \mathrm{p}=0.003 ; \mathrm{p}=0.89$ for interaction). In contrast, elevated BMI in both adolescence $(\beta=1.355, \mathrm{p}=0.004)$ and adulthood $(\beta=$ $1.207, \mathrm{p}=0.03)$ were independently associated with angiography-proven coronary heart disease $(\mathrm{p}=$ 0.048 for interaction). 
Conclusions: An elevated BMI in adolescence, one that is well within the range currently considered to be normal, constitutes a substantial risk factor for obesity-related disorders in midlife. Although the risk of diabetes is mainly associated with increased BMI close to the time of diagnosis, the risk of coronary heart disease is associated with an elevated BMI both in adolescence and in adulthood, supporting the hypothesis that the processes causing incident coronary heart disease, particularly atherosclerosis, are more gradual than those resulting in incident diabetes.

The aim of this study was to determine the impact of elevated BMI in adolescence and the increase of BMI during the transition from adolescence to adulthood on the development of T2DM and coronary heart disease in young adulthood. Data from the Israel Defense Force Medical Corps on all males remaining in the army and examined every 3-5 years were analyzed. Of 37,674 males followed for a mean of $17 \pm 7.4$ years, 1,173 developed T2DM and 327 angiography-proven coronary heart disease between ages 25 and 45 years. The important conclusions of this study are: (1) The development of T2DM in males in young adulthood is influenced mainly by their recent BMI and weight gain. Thus, an adolescent male with a relatively high $\mathrm{BMI}$, who grows up to become a lean adult, practically eliminates the risk of developing diabetes. (2) The development of coronary heart disease in males in young adulthood is independently influenced by both elevated BMI in adolescence and recent BMI, i.e. an elevated BMI in adolescent males predicts coronary heart disease in early adulthood, independent of a more recent BMI value. (3) The above refers to BMI values within what is considered the normal range, i.e. adolescent BMIs at the 49th percentile.

The authors suggested that clinical coronary heart disease in midlife is probably the consequence of gradually increasing atherosclerosis during adolescence and early adulthood, and that interventions during childhood to promote healthy lifestyle habits are essential for effective prevention of earlyonset heart disease in adulthood. I look forward to similar investigations about females.

\section{New mechanisms}

\section{The inflammasome-mediated caspase-1 activation controls adipocyte differentiation and insulin sensitivity}

Stienstra R, Joosten LA, Koenen T, van Tits B, van Diepen JA, van den Berg SA, Rensen PC, Voshol PJ, Fantuzzi G, Hijmans A, Kersten S, Muller M, van den Berg WB, van Rooijen N, Wabitsch M, Kullberg BJ, van der Meer JW, Kanneganti T, Tack CJ, Netea MG

Department of Medicine, Radboud University Nijmegen Medical Centre and Nijmegen Institute for Infection, Inflammation and Immunity, Nijmegen, The Netherlands.

r.stienstra@aig.umcn.nl

Cell Metab 2010;12:593-605.

Background: Obesity-induced inflammation originating from expanding adipose tissue interferes with insulin sensitivity. Important metabolic effects have been recently attributed to IL-1 $\beta$ and IL-18, two members of the IL- 1 family of cytokines. Processing of IL-1 $\beta$ and IL-18 requires cleavage by caspase-1, a cysteine protease regulated by a protein complex called the inflammasome. It was demonstrated that the inflammasome/caspase- 1 governs adipocyte differentiation and insulin sensitivity. Caspase- 1 is upregulated during adipocyte differentiation and directs adipocytes toward a more insulin-resistant phenotype.

Methods and Results: Treatment of differentiating adipocytes with recombinant IL-1 $\beta$ and IL-18, or blocking their effects by inhibitors, reveals that the effects of caspase- 1 on adipocyte differentiation are largely conveyed by IL-1 $\beta$. Caspase- 1 and IL- $1 \beta$ activity in adipose tissue is increased both in diet-induced and genetically induced obese animal models. Conversely, mice deficient in caspase- 1 are more insulin-sensitive as compared to wild-type animals. In addition, differentiation of preadipocytes isolated from caspase $-1^{-/}$or $\mathrm{NLRP}^{-/-}$mice resulted in more metabolically active fat cells. In vivo, treatment of obese mice with a caspase- 1 inhibitor significantly increases their insulin sensitivity. Indirect calorimetry analysis revealed higher fat oxidation rates in caspase- $1^{-/}$animals. 
Conclusions: The inflammasome is an important regulator of adipocyte function and insulin sensitivity, and caspase- 1 inhibition may represent a novel therapeutic target in clinical conditions associated with obesity and insulin resistance.

\section{The NLRP3 inflammasome instigates obesity-induced inflammation and insulin resistance}

Vandanmagsar B, Youm YH, Ravussin A, Galgani JE, Stadler K, Mynatt RL, Ravussin E, Stephens JM, Dixit VD Laboratory of Neuroendocrine-Immunology, Louisiana State University, Baton Rouge, La., USA.

Nat Med 2011;17:179-88.

Background: The emergence of chronic inflammation during obesity in the absence of overt infection or well-defined autoimmune processes is a puzzling phenomenon. The Nod-like receptor (NLR) family of innate immune cell sensors, such as the nucleotide-binding domain, leucine-rich-containing family, pyrin domain-containing-3 (Nlrp3, but also known as Nalp3 or cryopyrin) inflammasome are implicated in recognizing certain non-microbial originated 'danger signals' leading to caspase- 1 activation and subsequent interleukin-1 $(\mathrm{IL}-1 \beta)$ and IL-18 secretion.

Methods: The effect of calorie restriction and exercise-mediated weight loss in obese individuals with T2DM was studied.

Results: It was demonstrated that calorie restriction and exercise-mediated weight loss in obese individuals with T2DM is associated with a reduction in adipose tissue expression of Nlrp3 as well as with decreased inflammation and improved insulin sensitivity. In addition it was found that the Nlrp3 inflammasome senses lipotoxicity-associated increases in intracellular ceramide to induce caspase-1 cleavage in macrophages and adipose tissue. Ablation of Nlrp3 in mice prevents obesity-induced inflammasome activation in fat depots and liver as well as enhances insulin signaling. Furthermore, elimination of Nlrp3 in obese mice reduces IL-18 and adipose tissue interferon- $\gamma$ (IFN- $\gamma$ ) expression, increases naive T-cell numbers and reduces effector T-cell numbers in adipose tissue.

Conclusions: Data show that the Nlrp3 inflammasome senses obesity-associated danger signals and contributes to obesity-induced inflammation and insulin resistance.

These two articles elucidate the link between obesity, insulin resistance, and the chronic inflammation state. This pathophysiological condition, termed meta-inflammation, is characterized by abnormal cytokine production, increased acute-phase reactants, and the activation of a network of inflammatory signaling pathways. Both this pathophysiological state and insulin resistance are associated with the activation of macrophages in fat depots. Emerging evidence suggests that macrophages can recognize not only infectious agents but also various danger-associated molecular patterns (DAMPs), and subsequently release proinflammatory cytokines such as IL-1 $\beta$ and IL-18.

For the release of bioactive IL-1 $\beta$ and IL-18 from macrophages, a few essential steps are needed: (1) the processing of IL-1 $\beta$ and IL-18 by an intracellular cysteine protease called caspase-1; (2) the activation of caspase- 1 itself, which occurs by conformational changes, and (3) the formation of a multiprotein called inflammasome, wherein caspase- 1 undergoes conformational change required for its cleavage and full activation. NIrp3 is an inflammasome that can be activated by non-microbial-originated DAMPs such as extracellular ATP, urate crystals, asbestos, silica, and $\beta$-amyloid.

Stienstra et al. showed that caspase-1 expression increased during adipogenesis. In mice fed a highfat diet the expression of caspase-1 in adipose tissue increased in parallel to their weight gain. This was accompanied by an increase in IL-1 $\beta$ and IL-18. Blockage of caspase- 1 in human adipose tissue reduced the expression of IL-1 $\beta$. Finally, in caspase-1-deficient mice insulin signaling was improved. Vandanmagsar et al. demonstrated in mice a correlation between mRNA expression of II1 b and NIrp3 in visceral adipose tissue and both body weight and adiposity. Chronic calorie restriction resulted in significant reduction in NIrp3 and II1 b mRNA in both visceral and subcutaneous adipose tissue compared to that in normally fed mice. The clinical relevance of these data was investigated in obese individuals with T2DM before and after weight loss achieved by calorie restriction and exercise. Weight loss in obese individuals with T2DM resulted in substantial reduction in fat cell size and improvement of insulin sensitivity. As in mice, this was associated with a significant reduction in IL-1 $\beta$ and NLRP3 mRNA expression in subcutaneous adipose tissue. Furthermore, knockout of Nlrp3 resulted in reduction in caspase-1 activation in the liver, as well as improvement in insulin signaling in the liver, and reduced hepatic steatosis. Emerging from the above is a substantial role for Nlrp3 inflam- 
masome in sensing obesity-associated inducers of caspase-1 activation, and thereby regulating the development and the magnitude of inflammation and its downstream effects on insulin signaling. These findings are important since intervention in this cascade can be a target to treat obesity. Indeed mice treated with caspase-1 inhibitor showed significant improvement in insulin sensitivity. However, in a recent randomized, double-blind, crossover study, inflammation was lower in obese individuals with the metabolic syndrome treated with Anakinra - a natural IL-1 receptor antagonist than in those treated with a placebo. However, insulin sensitivity did not differ between the treatment groups [6]. Thus, until further investigations are performed, we still need to watch our weight and eat less.

\section{Metabolic syndrome}

\section{Mechanism of the year}

\section{Identification of an imprinted master trans-regulator at the KLF14 locus related to multiple metabolic phenotypes}

Small KS, Hedman AK, Grundberg E, Nica AC, Thorleifsson G, Kong A, Thorsteindottir U, Shin SY, Richards HB, Soranzo N, Ahmadi KR, Lindgren CM, Stefansson K, Dermitzakis ET, Deloukas P, Spector TD, McCarthy MI Department of Twin Research and Genetic Epidemiology, King's College London, London; Wellcome Trust Sanger Institute, Wellcome Trust Genome Campus, Hinxton, UK.

Nat Genet 2011;43:561-4.

Background: Genome-wide association studies have identified many genetic variants associated with complex traits. However, only a minority of loci have the molecular mechanisms mediating these associations been characterized. In parallel, whereas cis-regulatory patterns of gene expression have been extensively explored, the identification of trans-regulatory effects in humans has attracted less attention.

Results: The type 2 diabetes and high-density lipoprotein cholesterol-associated cis-acting expression quantitative trait locus (eQTL) of the maternally expressed transcription factor KLF14 acts as a master trans-regulator of adipose gene expression. Expression levels of genes regulated by this trans-eQTL are highly correlated with concurrently measured metabolic traits, and a subset of the trans-regulated genes harbor variants directly associated with metabolic phenotypes.

Conclusions: This trans-eQTL network provides a mechanistic understanding of the effect of the KLF14 locus on metabolic disease risk and offers a potential model for other complex traits.

Most phenotypic characteristics result from the interaction of multiple genes. Variation in gene expression contributes to both phenotypic diversity and to differences in disease susceptibility.

Expression quantitative trait loci (eQTLs) are genomic loci that regulate gene transcription and expression on a genome-wide scale. The control of gene expression is through complex countless regulatory genes and regulatory proteins. Cis-regulatory elements are present on the same molecule of DNA as the gene they regulate, whereas trans-regulatory elements may modify the expression of genes distant from the gene from which they were transcribed.

The Krüppel-like factor (KLF) is a family of transcription factors. Thus far, 17 members of the KLF family have been identified and characterized, 7 with definite roles in adipogenesis. Variants near the transcription factor KLF1 have previously been associated with T2D and HDL-C in large gene-wide association study analyses. In the current study, the authors demonstrated that KLF14 influenced in both in cis eQTL and trans patterns simultaneously. Only the maternally expressed transcription factor KLF14 acts (imprinting). Cis-acting influence of the development of T2DM and HDL-C while on trans-acting KLF14 acts as a 'master regulator' of a network of other genes in adipose tissue. This trans-acting is highly correlated with measures of the metabolic syndrome, i.e. BMI, triglycerides, fasting glucose and insulin, HOMA-IR, adiponectin, and waist circumference. This trans-eQTL network provides a potential model for other complex traits. 


\section{Insulin resistance in children: consensus, perspective, and future directions}

Levy-Marchal C, Arslanian S, Cutfield W, Sinaiko A, Druet C, Marcovecchio ML, Chiarelli F

Institut National de la Santé et de la Recherche Médicale, Unité 690, Hôpital Robert Debré, Université Paris Diderot, Paris, France.

J Clin Endocrinol Metab 2010;95:5189-98.

Background: Emerging data indicate that insulin resistance is common among children and adolescents and is related to cardiometabolic risk, therefore requiring consideration early in life. However, there is still confusion on how to define insulin resistance, how to measure it, what its risk factors are, and whether there are effective strategies to prevent and treat it. A consensus conference was organized in order to clarify these points.

Methods: The consensus was internationally supported by all the major scientific societies in pediatric endocrinology and 37 participants. An independent and systematic search of the literature was conducted to identify key articles relating to insulin resistance in children. The conference was divided into five themes and working groups: background and definition; methods of measurement and screening; risk factors and consequences; prevention, and treatment. Each group selected key issues, searched the literature, and developed a draft document. During a 3-day meeting, these papers were debated and finalized by each group before presenting them to the full forum for further discussion and agreement. Results and Conclusions: Given the current childhood obesity epidemic, insulin resistance in children is an important issue confronting healthcare professionals. There are no clear criteria to define insulin resistance in children, and surrogate markers such as fasting insulin are poor measures of insulin sensitivity. Based on current screening criteria and methodology, there is no justification for screening children for insulin resistance. Lifestyle interventions including diet and exercise can improve insulin sensitivity, whereas drugs should be implemented only in selected cases.

This is a timely consensus. During the last year about 250 articles about metabolic syndrome in children were published. Determination of the best means of assessing insulin resistance in children is needed, especially since this parameter serves in many studies as an important factor for the definition and long-term evaluation of the metabolic syndrome.

The major points in the consensus are that there are currently no standards for insulin resistance in children, and no definitions for normal and abnormal levels. As the authors state, this is due to the use of different techniques in measuring insulin sensitivity, small cohort sizes to establish normative distribution, and a lack of long-term outcomes. Fasting insulin is evidently not an optimal tool for assessing peripheral insulin sensitivity in children. Therefore, the committee concluded that until separate standards for gender, ethnic groups, and pubertal stages are developed, there is no recommendation for insulin measurement. The consensus also reviewed risk factors for developing insulin resistance, their consequences and treatment modalities.

Follow-up on a Yearbook 2009 paper

\section{One-year follow-up of untreated obese white children and adolescents with impaired glucose tolerance: high conversion rate to normal glucose tolerance}

Kleber M, Lass N, Papcke S, Wabitsch M, Reinehr T

Vestische Hospital for Children and Adolescents, University of Witten/Herdecke, Ulm, Germany.

Diabet Med 2010;27:516-21.

Background: Impaired glucose tolerance (IGT) is regarded at risk factor for later diabetes. The authors aimed to identify predictive factors for outcome of IGT in obese children and adolescents. 
Methods: 79 obese white children and adolescents (mean age $13.1 \pm 2.1$ years, $51 \%$ female, $76 \%$ pubertal) with IGT were prospectively examined. Anthropometrics, 2 h glucose in oral glucose tolerance test (OGTT), fasting glucose, insulin, insulin resistance index homeostasis model assessment (HOMA), glycated hemoglobin (HbA1c)), lipids, blood pressure, waist circumference and pubertal stage were determined at baseline and 1 year later.

Results: At follow-up, 32\% of the children continued to have IGT, 66\% converted to normal glucose metabolism, 1 child had impaired fasting glucose and 1 child developed T2DM. Children with improvement of IGT had significantly lower weight, waist circumference, triglycerides, $2 \mathrm{~h}$ glucose during OGTT and HbA1c at baseline compared with children who continued to have IGT. In the children whose glucose tolerance became normal, weight fell, and serum insulin concentrations, HOMA, lipids and blood pressure improved. They were also more likely to enter the late or postpubertal stage than children who continued to have IGT.

Conclusions: Predictive factors for the frequent normalization of IGT in obese children and adolescents were lower weight, HbA1c and $2 \mathrm{~h}$ glucose levels in OGTT at baseline, as well as a reduction of weight and entering late puberty stages during follow-up. Cardiovascular risk factors and HOMA improved along with the improvement of IGT, supporting an association between IGT, insulin resistance and features of the metabolic syndrome.

This study was conducted in a center specializing in pediatric obesity, where all obese subjects are evaluated according to the guidelines of the German Pediatric Obesity Association and are screened for overweight, hypertension, dyslipidemia, and impaired glucose metabolism. The investigators followed 79 obese children with impaired glucose tolerance (IGT) who chose not to participate in a lifestyle intervention program. At 1-year follow-up, $66 \%$ of the children converted to normal glucose metabolism; IGT persisted in one third of the children. Those diagnosed with IGT at 1-year follow-up were heavier, and with central obesity, higher glucose levels, as measured by the OGTT, and higher triglyceride levels. However, there was no difference between those who continued to have impaired IGT and those who converted to normal glucose test in age, blood pressure, LDL cholesterol, and, coincident with the consensus on insulin resistance (above), with fasting insulin values, insulin concentrations, or insulin resistance index HOMA.

In the study by Libman et al. presented in the 2009 Yearbook [7], only $30 \%$ of obese adolescents who were identified with IGT showed abnormal results in a test repeated 1 month later. This suggests poor reproducibility of the IGT test. The current study reinforces the problematic validity of the OGTT. The high conversion rate of IGT to normal glucose tolerance in obese white children in the course of 1 year challenges the clinical relevance of IGT in obese children and adolescents, and the relevance of the definitions of IGT to adolescents. Since OGTT is labor-intensive, with apparently poor reproducibility among obese adolescents, both in the short and long term, its widespread use should be questioned.

\section{Clinical trials}

\section{A school-based intervention for diabetes risk reduction}

Foster GD, Linder B, Baranowski T, Cooper DM, Goldberg L, Harrell JS, Kaufman F, Marcus MD, Trevino RP, Hirst K The HEALTHY Study Group

N Engl J Med 2010;363:443-53.

Background: The effects of a multicomponent, school-based program addressing risk factors for diabetes among children whose race or ethnic group and socioeconomic status placed them at high risk for obesity and T2DM were examined.

Methods: Using a cluster design, 42 schools were randomly assigned to either a multicomponent schoolbased intervention (21 schools) or assessment only (control, 21 schools). A total of 4,603 students participated (mean \pm SD age, $11.3 \pm 0.6$ years; $54.2 \%$ Hispanic and $18.0 \%$ Black; $52.7 \%$ girls). At the beginning of sixth grade and at the end of eighth grade, students underwent measurements of body mass index (BMI), waist circumference, and fasting glucose and insulin levels. 
Results: There was a decrease in the combined prevalence of overweight and obesity, in both the intervention and control schools, with no significant difference between the school groups. The intervention schools had greater reductions in the secondary outcomes of BMI z score, percentage of students with waist circumference at or above the 90th percentile, fasting insulin levels ( $\mathrm{p}=0.04$ for all comparisons), and prevalence of obesity $(\mathrm{p}=0.05)$. Similar findings were observed among students who were at or above the 85 th percentile for BMI at baseline. Less than $3 \%$ of the students who were screened had an adverse event; the proportions were nearly equivalent in the intervention and control schools.

Conclusions: A comprehensive school-based program did not result in greater decreases in the combined prevalence of overweight and obesity than those that occurred in control schools. However, the intervention did result in significantly greater reductions in various indexes of adiposity. These changes may reduce the risk of childhood-onset T2DM.

In light of the dramatic increase in childhood obesity, means are needed to reduce secondary morbidity. School-based intervention programs seem a natural place for the promotion of physical activity and healthy eating. The results of this study, aimed to evaluate the effects of a 3-year multicomponent school-based program on the prevalence of overweight and obesity, are therefore important. In a large randomized intervention study conducted, about half of the 4,603 sixth grade participants were overweight or obese at the beginning of the study. The intervention consisted of four components: nutrition, physical activity, behavioral knowledge, and communication skills. I encourage you to browse the intervention material at www.healthystudy.org. Without a doubt, this is a comprehensive program involving school staff, parents, and students, and thoroughly addressing many components of healthy behavior.

After 3 years of intervention, a $16.5 \%$ decrease in the prevalence of overweight and obesity was observed in the schools in which the intervention was implemented, and a $15.9 \%$ decrease in the control schools. Considering the tremendous investment in planning and operating the program, these findings are extremely disappointing. In general, most previous school-based studies were successful in achieving specific goals, such as improved eating habits, knowledge acquisition, or increased physical activity, but rarely had an impact on obesity.

We should not give up. The impact of an educational program may become evident only in a number of years. Further, education of young parents could reflect positively on younger siblings. But is it possible that intervention at school age is already too late? Should efforts be aimed toward preschool and kindergarten children? Also, are schools not powerful enough for such a big change? We are awaiting publications of the results of the EPODE and 'Tiger Kids' programs, two innovative community projects to promote healthy behaviors among children. The Tiger Kids is aimed at both preschool and school-aged children in four regions of Germany and the EPODE brings together parents, teachers, health professionals, and the local business community in towns across France. Hopefully, targeting younger age groups and all stakeholders in the community will yield better results.

\title{
Non-high-density lipoprotein cholesterol concentration is associated with the metabolic syndrome among US youth aged 12-19 years
}

\author{
Li C, Ford ES, McBride PE, Kwiterovich PO, McCrindle BW, Gidding SS \\ Division of Adult and Community Health, National Center for Chronic Disease Prevention and Health Promotion, \\ Centers for Disease Control and Prevention, Atlanta, Ga., USA. \\ cli@cdc.gov \\ J Pediatr 2011;158:201-7.
}

Background: To test the hypothesis that the concentration of non-high-density lipoprotein cholesterol (non-HDL-C) is associated with the metabolic syndrome (MetS) in youth.

Methods: Data on 2,734 children and adolescents aged 12-19 years from the cross-sectional National Health and Nutrition Examination Survey 1999-2004 were analyzed.

Results: Depending on the definition of MetS used, the mean non-HDL-C concentration among youth with MetS ranged from 144.2 to $155.8 \mathrm{mg} / \mathrm{dl}$, compared with $108.8-109.1 \mathrm{mg} / \mathrm{dl}$ in those without MetS (all p < 0.001). The MetS prevalence ranged from 6.9 to $11.7 \%$ in youth with a non-HDL-C concentration of $120-144 \mathrm{mg} / \mathrm{dl}$ and from 21.5 to $23.4 \%$ in those with a concentration $\geq 145 \mathrm{mg} / \mathrm{dl}-$ both significantly higher than the prevalence of $1.9-3.4 \%$ in youth with a concentration $<120 \mathrm{mg} / \mathrm{dl}$ (all $\mathrm{p}<0.001)$. After adjustment for potential confounders, youth with a non-HDL-C concentration 
$\geq 120$ or $\geq 145 \mathrm{mg} / \mathrm{dl}$ were about 3 or 4 times more likely to have MetS compared with those with a non-HDL-C $<120$ or $<145 \mathrm{mg} / \mathrm{dl}$ (all $\mathrm{p}<0.001$ ).

Conclusions: Fasting non-HDL-C concentration was strongly associated with MetS in US youth. The authors recommend the use of non-HDL-C thresholds of 120 and $145 \mathrm{mg} / \mathrm{dl}$ to indicate borderline and high MetS risk, respectively.

Non-HDL-C concentration is measured by subtracting the HDL-C concentration from the total serum cholesterol concentration. The non-HDL-C value reflects the concentration of many atherogenic lipoproteins, including LDL-C, very-low-density lipoprotein cholesterol (VLDL-C), intermediate-density lipoprotein cholesterol (IDLC), and lipoprotein(a). Among adults, non-HDL-C concentration is an important predictor of premature atherosclerosis and first myocardial infarction. The current criteria of the metabolic syndrome (MetS) include only triglyceride and HDL levels, basically ignoring the impact of LDL and other atherogenic lipoproteins. This study tested the hypothesis that non-HDL concentration is associated with the MetS among US youth aged 12-19 years. Because of the lack of an accepted pediatric definition of MetS in childhood, four definitions of MetS were used in this study: The overall mean concentration of non-HDL-C was $111.7 \mathrm{mg} / \mathrm{dl}$, and the median concentration was $107.8 \mathrm{mg} / \mathrm{dl}$. The mean non-HDL-C concentration was similar between males and females. Based on the prevalence of MetS according to non-HDL-C concentration, the authors suggested the use of non-HDL-C threshold values of $120 \mathrm{mg} / \mathrm{dl}$ to indicate borderline risk, and $145 \mathrm{mg} / \mathrm{dl}$ to indicate high risk for the MetS. The association between non-HDL-C and MetS may be explained through the obesity-inflammation-insulin resistance linkage, as well as through atherogenic processes.

Non-HDL cholesterol can be assessed in patients with triglyceride levels $>400 \mathrm{mg} / \mathrm{dl}$ and in patients who are not fasting. If non-HDL is a better predictor of cardiovascular disease than LDL, perhaps it should be used as a criterion for the determination of the metabolic syndrome.

\section{Dyslipidemia}

\section{Concepts revised}

\section{Universal versus targeted blood cholesterol screening among youth: the CARDIAC Project}

Ritchie SK, Murphy EC, Ice C, Cottrell LA, Minor V, Elliott E, Neal W

Department of Pediatrics, Robert C. Byrd Health Science Center, West Virginia University, Morgantown, W.Va., USA.

sritchie@hsc.wvu.edu

Pediatrics 2010;126:260-5

Background: To determine the sensitivity and specificity of family history in identifying children with severe or genetic hyperlipidemias in a rural, predominantly white population.

Methods: A total of 20,266 fifth-grade children in West Virginia, from the Coronary Artery Risk Detection in Appalachian Communities (CARDIAC) Project who completed a family history and fasting lipid profile, were used in analyses. The relationship between hyperlipidemia and family history was determined, and the use of family history to predict the need for pharmacologic treatment among children with dyslipidemia was evaluated.

Results: A total of $71.4 \%$ of children met the National Cholesterol Education Program (NCEP) guidelines for cholesterol screening on the basis of positive family history. Of those, 1,204 (8.3\%) were considered to have dyslipidemia ( $\mathrm{LDL} \geq 130 \mathrm{mg} / \mathrm{dl}$ ), and $1.2 \%$ of these children with dyslipidemia warranted possible pharmacologic treatment (LDL $\geq 160 \mathrm{mg} / \mathrm{dl}$ ). Of the $28.6 \%$ who did not have a positive family history (did not meet NCEP guidelines), 548 (9.5\%) had dyslipidemia, 1.7\% of whom warranted pharmacologic treatment. Sensitivity and specificity data demonstrated that family history does not provide a strong indication as to whether pharmacologic treatment may be warranted.

Conclusions: Results indicate that the use of family history to determine the need for cholesterol screening in children would have (1) missed many with moderate dyslipidemia and (2) failed to detect a substantial number with likely genetic dyslipidemias that would require pharmacologic treatment. The use of 
universal cholesterol screening would identify all children with severe dyslipidemia, allowing for proper intervention and follow-up and leading to the prevention of future atherosclerotic disease.

Daniel Kahneman, Laureates Prize in Economic Sciences in Memory of Alfred Nobel 2002, relayed the discovery of his 'first cognitive illusion'. Serving as a psychologist during his service in the Israel Defense Forces, he participated in the assessment of candidates for officer training. One test, developed by the British Army in the Second World War, involved a group dynamics exercise. Two psychologists observed the candidates, looking for leadership traits. It was felt that the stress of the exercise would reveal the soldiers' true nature, and enable identification of those who would be good leaders and those who would not. The psychologists received periodic feedback from the officer-training school, indicating the accuracy of their ratings of candidates' potential. The story was always the same: their prediction of capable officers was unreliable. Nevertheless, the psychologists continued to use the same tests and measures over and over. The complete lack of connection between the statistical information and the compelling experience of insight was so impressive that Prof. Kahneman coined a term for it: 'the illusion of validity' [8].

This story is inspiring and of particular relevance considering recommendations for lipid screening in children. Two important conclusions can be drawn from the current study. Firstly, more than two thirds of the children met the National Cholesterol Education Program (NCEP) guidelines for cholesterol screening on the basis of positive family history! Secondly, it was clearly demonstrated that the selective screening according to the current guidelines would have missed many children who are at risk for atherosclerosis and for whom timely intervention may prevent or delay cardiovascular disease. This year the National Lipid Association Expert Panel on Familial Hypercholesterolemia [9] recommended universal screening at age 9-11 years for all children, as well as earlier screening beginning at age 2 years for children with a family history of premature cardiovascular disease. I hope these recommendations will be adopted by the American Academy of Pediatrics as well.

\section{Important for clinical practice}

\section{Changes and variability in high levels of low-density lipoprotein cholesterol among children}

Freedman DS, Wang YC, Dietz WH, Xu JH, Srinivasan SR, Berenson GS

Division of Nutrition, Physical Activity, and Obesity, Centers for Disease Control and Prevention, Atlanta, Ga., USA. dxf1@cdc.gov

Pediatrics 2010;126:266-73.

Background: A 2008 report from the American Academy of Pediatrics recommended both population and individual approaches (including pharmacologic interventions) for adolescents who had low-density lipoprotein (LDL) cholesterol levels above various cutoff points (130, 160, and $190 \mathrm{mg} / \mathrm{dl})$. However, the tracking and variability of these very high levels have not been investigated.

Methods: A total of 6,827 subjects underwent multiple LDL cholesterol determinations in childhood and adulthood in the Bogalusa Heart Study. The total number of determinations was 26,748, and the median interval between examinations was 3 years.

Results: Correlations between initial and subsequent LDL cholesterol levels ranged from approximately 0.8 for measurements made within the same year to approximately 0.5 for periods of $>$ or $=20$ years. Most children who had very high LDL cholesterol levels, however, had substantially lower levels at the next examination. LDL cholesterol levels between 160 and $189 \mathrm{mg} / \mathrm{dl}(\mathrm{n}=201)$ decreased, on average, by $21 \mathrm{mg} / \mathrm{dl}$ at the next examination, whereas levels of $>$ or $=190 \mathrm{mg} / \mathrm{dl}(\mathrm{n}=44)$ decreased by $34 \mathrm{mg} /$ dl. In contrast, the mean increase for LDL cholesterol levels of $<70 \mathrm{mg} / \mathrm{dl}$ was $13 \mathrm{mg} / \mathrm{dl}$. These changes were equal to those expected on the basis of regression to the mean.

Conclusions: There can be large changes in extreme levels of LDL cholesterol because of regression to the mean, and practitioners should be aware that very high levels may decrease substantially in the absence of any intervention. 
If population screening will indeed be implemented, we learn from the current study that it will be important to interpret the results with caution. The aim of this study was to track the variability of abnormal LDL levels in children and adolescents over a number of years. Participants were 6,827 subjects, who were examined an average of 5 times (range 2-13 times) with a median period between examinations of about 3 years. The authors found that despite a moderately strong tracking of LDL cholesterol levels, children with very high levels ( $\geq 160 \mathrm{mg} / \mathrm{dl}$ ) were likely to have substantially lower levels (mean decreases of 20-30 mg/dl) at re-examination. Changes in LDL cholesterol levels between examinations were great enough to alter the recommended treatment in many children.

Although this is important practical data, one should remember that a natural and expected decrease occurs in LDL cholesterol levels during puberty. Thus, the results may partially reflect this trend, as well as biological variabilities. Since the current recommendation for treating children with elevated LDL levels are to start medications only after 6 months of diet, repeated blood test should be performed, in any case, within a reasonable time. The authors concluded that after identifying subjects with suspected hyperlipidemia, at least two or three repeated measurements are needed to further characterize their actual lipid/lipoprotein profile. I totally agree with that.

\section{Reviews}

\section{Statins for children with familial hypercholesterolemia}

Vuorio A, Kuoppala J, Kovanen PT, Humphries SE, Strandberg T, Tonstad S, Gylling H Mehilainen Airport Medical Center, Vantaa, Finland.

Cochrane Database Syst Rev 2010:CD006401.

Background: Familial hypercholesterolemia is one of the most common inherited metabolic diseases; the average worldwide prevalence of heterozygous familial hypercholesterolemia is about 1 in 500 . Diagnosis of familial hypercholesterolemia in children is based on two measurements of low-density lipoprotein cholesterol levels $>4.0 \mathrm{mmol} / \mathrm{l}$ or a DNA-based analysis. Coronary stenosis has been detected in men with familial hypercholesterolemia as young as 17 years old and in women with familial hypercholesterolemia at 25 years old. Atherosclerosis and its clinical complications occur prematurely, especially in men, thus lifelong hypolipidemic measures, started in childhood, are needed to reduce the risk of cardiovascular diseases. In children with familial hypercholesterolemia children, so far diet has been the main mode of treatment. Anion exchange resins, such as cholestyramine and colestipol, have also been found to be effective but are generally considered unpalatable and therefore poorly tolerated. Since the 1990s, statin trials have been carried out among children with familial hypercholesterolemia (aged 7-17 years), and statins reduced their serum low-density lipoprotein cholesterol levels by $23-40 \%$. The safety of statins among children is not well known even though statins seem to be safe and well tolerated in adults.

Objectives: To assess the effectiveness and safety of statins in children with familial hypercholesterolemia. Relevant trials were identified from the Group's Inborn Errors and Metabolism Trials Register and Medline. Randomized and controlled clinical trials including participants up to 18 years of age compared statins to placebo or to diet alone.

Results: 19 potentially eligible studies were found of which eight randomized placebo-controlled trials (897 participants) were included. Statins reduced the mean low-density lipoprotein cholesterol LDL-C concentration at all time points. There was no difference between serum aspartate and alanine aminotransferase as well as creatine kinase concentrations at any time-point. The risks of myopathy and clinical adverse events were also similar in both groups. In one study, simvastatin was shown to improve flow-mediated dilation of the brachial artery.

Conclusions: Statin treatment is an efficient lipid-lowering therapy in children with familial hypercholesterolemia. It seems to be safe in the short term, but long-term safety is unknown. Children treated with statins should be carefully followed up by their pediatricians. Large long-term randomized controlled trials are needed to establish the long-term safety of statins. 
The Cochrane Collaboration is named in honor of Archie Cochrane, a medical researcher born in Scotland (ESPE 2011!) who contributed greatly to the development of epidemiology as a science. Cochrane stressed the importance of evidence from randomized controlled trials (RCTs) as a reliable information source. He wrote, 'It is surely a great criticism of our profession that we have not organized a critical summary, by specialty or subspecialty, adapted periodically, of all relevant randomized controlled trials.' His challenge eventually led to the opening of The Cochrane Collaboration - an international network of people helping healthcare providers, policymakers, patients, their advocates and caregivers make well-informed decisions about human healthcare by preparing, updating, and promoting the accessibility of Cochrane Reviews.

The objective of the current review was to assess the effectiveness and safety associated with the use of statins in children heterozygous for familial hypercholesterolemia. Since the incidence of and mortality from cardiovascular diseases in children are very rare, the following surrogate end-points for effectiveness were used: (1) change in carotid intima-media thickness, (2) change in serum LDL cholesterol level, and (3) change in measures of growth and maturation. In addition, adverse events associated with statin treatment, such as liver dysfunction, myopathy, myotoxicity and myocardial infarction, were studied. The age of the study participants ranged from 4 to 18 years, $58 \%$ were males. In general, the intervention and follow-up time was short, ranging from 6 weeks to 2 years. The pooled estimate of the difference in mean relative reductions of LDL levels at the end of the follow-up was $-32.15 \%$. It is reassuring to know that there was no difference between the number of participants treated with statins and those in the control group presenting with a clinically significant increase in liver enzymes, in creatine kinase values, in myopathy, and in sexual maturation. The authors concluded that the putative risk of statin toxicity in children is outweighed by the reduced cardiovascular risk achieved by statin treatment, and that larger and longer-term RCTs are needed to ensure that statins are safe in long-term therapy.

\section{New concerns}

\section{Dyslipidemia of mothers with familial hypercholesterolemia deteriorates lipids in adult offspring}

Van der Graaf A, Vissers MN, Gaudet D, Brisson D, Sivapalaratnam S, Roseboom TJ, Jansen AC, Kastelein JJ, Hutten BA

Department of Vascular Medicine, Academic Medical Centre, Amsterdam, The Netherlands.

Arterioscler Thromb Vasc Biol 2010;30:2673-7.

Background: It is unknown whether elevated maternal LDL-C levels lead to dyslipidemia in the offspring. Because this could have important consequences for cardiovascular prevention in mother and child, we explored the relationship between maternal familial hypercholesterolemia (FH) and lipids in adult offspring.

Methods: In a large cohort of both Dutch and Canadian origin, lipid profiles between patients, aged 18-85 years, who inherited FH maternally $(\mathrm{n}=1,069)$ and those who inherited FH paternally $(\mathrm{n}=$ 1,270 ) were compared. This relationship was evaluated using multivariate regression analyses.

Results: Levels of total cholesterol (TC), LDL-C, and apolipoprotein B 100 (ApoB100) were significantly elevated in patients who inherited $\mathrm{FH}$ maternally compared with patients who inherited $\mathrm{FH}$ paternally (adjusted differences in TC: $0.156 \mathrm{mmol} / \mathrm{l}, \mathrm{p}=0.037$; LDL-C: $0.187 \mathrm{mmol} / \mathrm{l}, \mathrm{p}=0.012$; ApoB: 0.064 $\mathrm{g} / \mathrm{l}, \mathrm{p}=0.022$ ).

Conclusions: The data show that maternal hereditary hypercholesterolemia slightly increases TC, LDL-C, and ApoB levels in their offspring later in life. Although the molecular mechanisms underlying these observations still require elucidation, our data suggest that maternal hypercholesterolemia during pregnancy may program lipid metabolism to a certain extent in the fetus.

Familial hypercholesterolemia $(\mathrm{FH})$ is a common autosomal dominant disorder of lipoprotein metabolism, caused by mutations in the low-density lipoprotein receptor gene. During pregnancy, females with $\mathrm{FH}$ discontinue statin therapy because of fear of potential teratogenicity. A growing body of 
evidence suggests that maternal hypercholesterolemia is associated with the development of fetal atherosclerosis. Studies in animal models show that the effect is not limited to the fetus but persists long after birth. Experiments in rabbits established that temporary diet-induced maternal hypercholesterolemia enhances both fetal lesion formation and the susceptibility to atherosclerosis later in life, supporting the assumption that hypercholsterolemia during pregnancy is associated with genetic programming.

The current study shows that adult offspring of $\mathrm{FH}$ mothers had slightly but statistically significant increased levels of TC, LDL-C, and ApoB compared with offspring of FH fathers.

Although the differences in these lipid levels are rather small, and their clinical relevance is not clear, the notion that environmental conditions can modulate the impact of genetic predisposition to hypercholesterolemia is fascinating.

The mechanisms underlying the long-term effects of maternal hypercholesterolemia in offspring are not clear. The authors suggest that epigenetic programming of metabolism during embryonal or fetal development might be involved.

\section{References}

1. Tjaden PG, Thoennes N: Extent, nature, and consequences of rape victimization: findings from the National Violence Against Women Survey. Washington, US Department of Justice, Office of Justice Programs, National Institute of Justice, 2006.

2. Noll JG, Zeller MH, Trickett PK, Putnam FW: Obesity risk for female victims of childhood sexual abuse: a prospective study. Pediatrics 2007;120:e61-e67.

3. Steen E, Terry BM, Rivera EJ, Cannon JL, Neely TR, Tavares R, Xu XJ, Wands JR, de la Monte SM: Impaired insulin and insulin-like growth factor expression and signaling mechanisms in Alzheimer's disease - is this type 3 diabetes? J Alzheimers Dis 2005; 7:63-80.

4. Hofman PL, Regan F, Jackson WE, Jefferies C, Knight DB, Robinson EM, Cutfield WS: Premature birth and later insulin resistance. N Engl J Med 2004;351:2179-2186.

5. Regan FM, Cutfield WS, Jefferies C, Robinson E, Hofman PL: The impact of early nutrition in premature infants on later childhood insulin sensitivity and growth. Pediatrics 2006;118:1943-1949.

6. Van Asseldonk EJ, Stienstra R, Koenen TB, Joosten LA, Netea MG, Tack CJ: Treatment with Anakinra improves disposition index but not insulin sensitivity in nondiabetic subjects with the metabolic syndrome: a randomized, double-blind, placebo-controlled study. J Clin Endocrinol Metab 2011;96:2119-2126.

7. Libman IM, Barinas-Mitchell E, Bartucci A, Robertson R, Arslanian S: Reproducibility of the oral glucose tolerance test in overweight children. J Clin Endocrinol Metab 2008;93:4231-4237.

8. Kahneman D, Tversky A: On the psychology of prediction. Psychol Rev 1973;80:237-251.

9. Goldberg AC, Hopkins PN, Toth PP, Ballantyne CM, Rader DJ, Robinson JG, Daniels SR, Gidding SS, de Ferranti SD, Ito MK, McGowan MP, Moriarty PM, Cromwell WC, Ross JL, Ziajka PE: Familial hypercholesterolemia: screening, diagnosis and management of pediatric and adult patients: clinical guidance from the National Lipid Association Expert Panel on Familial Hypercholesterolemia. J Clin Lipidol 2011;5:S1-S8. 